Breda Pogorelec

L.jubljana

\title{
DIE DEPENDENZGRAMMATIK VON TESNIÈRE UND DIE NEUE SLOWENISCHE SYNTAX
}

In der Enzyklopädie der slowenischen Sprache (Toporišič 1992: 159) wird die Dependenzgrammatik unter besonderem Stichwort erklärt, in dem diese Grammatik zuerst als "eine Grammatik, die vor allem die Dependenzrelationen im Satz untersucht", definiert wird. Dann werden laut Lucien Tesnière im Satz Reza poje drei Elemente dargestellt: Reza, poje + die Verbindung dazwischen und dann werden drei Stufen des Graphs mit dem Verb als "dem Element, das die Ganzheit zusammenhält" erklärt; außerdem wird gesagt, daß die Koordination gemäß dieser Theorie durch die entsprechende Konjunktion ausgedrückt wird (aus dem graphischen Schema ist ersichtlich, daß dabei die Satzteilkoordination gemeint ist: pojeta - Tine - in - Tone. Im Kommentar wird hinzugefügt, daß auch "unsere" (= moderne slowenische) Satzanalyse so aussieht, d. i. sie fängt mit dem Prädikat an, dann fragt man nach folgenden drei Hauptsatzteilen (Subjekt, Objekt, adverbiellen Prädikatbestimmungen), dann nach Attributen und deren Bestimmungen. Es wird die Methode des Unterstreichens von Satzteilen dargestellt, außerdem wird auf mehrere Arten der Dependenzgrammatik und auf verschiedene graphische Bezeichnungen der sprachlichen Erscheinungen aufgrund dieser Methode hingewiesen. - Im demselben Werk werden unter dem Stichwort translativ (Toporišič 1992: 331) zwei Bedeutungen gezeigt, von denen die erste auf einen entfernten Zusammenhang mit der Theorie von Tesnière hinweist, obwohl diese nicht erwähnt ist, lediglich bei der Definition selbst, nicht aber bei den Beispielen; translativ ist nach dieser Enzyklopädie "was aus einem Zustand in den anderen übertragen wird". Aber aus den Beispielen kann man sehen, das geht es um die Translation und nicht um Translativ als Instrument dieser Translation.

Die Dependenztheorie von Tesnière ist in der neueren slowenischen Syntax weder ausführlich dargestellt noch ausdrücklicher kritisch bewertet; eine Spur Kritik ist in erwähnten Artikel im Hinweis auf mehrere Dependenzgrammatiken oder auf den entsprechenden Graph zu sehen, nach dem das Subjekt nicht wie bei Tesnière dem Prädikat untergeordnet ist. Die zitierte Abhandlung in der Enzyklopädie der slowenischen Sprache zeigt jedoch, wie die Annahme dieser Theorie im slowenischen Raum von Teilinteresse der Forscher bei der Suche nach theoretischen Anregungen für eigene konkrete Aufgaben abhängt. 
Die neue slowenische Sprachwissenschaft kam in der Zeit nach 1955 mit dem damals aktuellen Strömungen in der Linguistik in Berührung, dies auch in Bereich der Syntax. Auf dem ersten internationalen Slavistentreffen nach dem zweiten Weltkrieg in Belgrad (1955) zeigte eine ganze Reihe hervorragender Sprachwissenschaftler aus der ganzen Welt neue Wege und Möglichkeiten auf und verwies auch auf die Errungenschaften und Tendenzen der slawistischen Sprachwissenschaft in der ersten Hälfte des 20. Jahrhunderts, besonders im ersten Nachkriegsjahrzehnte. Hätte es in der slowenischen linguistischen Wissenschaftszene einen lebendigeren und prompten Gedankenfluß gegeben, so hätten schon früher die sprachwissenschaftlichen Konzepte des slowenischen Romanisten R. F. Mikuš greifen können, die dieser bereits 1948 vorgestellt hatte. Dies insbesondere in der Polemik mit der zeitgenossischen serbischen Sprachwissenschaft im Jahre 1952. Doch unsere Generation hatte sich in Studium nicht mit der Syntax als besonderem Kapitel der (slowenistischen) Sprachwissenschaft befaßt, und war deßhalb nicht bereit eine solche Diskussion zu führen. Trotz einiger bemerkenswerter Arbeiten Anton Brezniks (+ 1943) war die Syntax in der historischen Grammatik kein Prioritätsbereich; das moderne Verständnis der synchronen Sprachwissenschaft als gleichberechtiges Feld der synchronen Sprachwissenschaft setzte sich nur mit Schwierigkeiten durch. Als dann die slowenische Syntax zu einem Gebiet geworden war, das es nun nur noch zu entwickeln galt, hielt Fran Ramovš in seinem letzten, nach seinem Tode publiziertem Bericht über die Arbeit des Institutes für slowenische Sprache der Slowenischen Akademie der Wissenschaften (1952) fest, daß in diesem Institut ein Mitarbeiter gebraucht werde, der sich mit Problemen der Syntax befassen würde und den man zur Weiterbildung nach Frankreich schicken müsse. Auf diesen Gedanken war Ramovš zweifellos angesichts der Syntagmatik des bereits erwähnten slowenischen Romanisten R. F. Mikuš gekommen, der sich damals als erstiger und einziger Slowene an der aktuellen Diskussion über die neue (strukturelle) Syntax beteiligte, die zu dieser Zeit auch L. Tesnière entwickelte. Ramovšs Pläne wurden durch seinen Tod zunichte gemacht, und zwar deshalb, weil man für die neue Sprachwissenschaft noch nicht offen war. Eine grössere Anzahl von Sprachwissenschaftlern hat erst seit Ende der fünfziger Jahre begonnen, sich mit der Syntax zu beschäftigen, sei es in einzelnen Themengebieten (Pogorelec, Toporišič, Orešnik, Dular, Križaj-Ortar, Kaučič-Baša und andere), sei es schon weitergehend in Zusammenhang mit einer gewissen Gesamtdarstellung in der slowenischen Grammatik (Toporišič); die Dependenztheorie verwendete auch Ada Vidovič Muha in der slowenischen Wortbildungslehre. Nicht zuletzt ist die Syntax als Gegenstand des Universitätstudiums der slowenischen Sprache erwähnenswert.

Ich selbst habe mich bereits frühzeitig mit Tesnière befaßt, zunächst in meiner Dissertation über die Konjunktion in der slowenischer Schriftsprache (1964). In diesem Themenzusammenhang waren für mich von besonderem Interesse die Kapitel über die Junktion und über die einfache und komplexe Translation, mehr noch als das einführende Kapitel: das heißt, die parataktische und hypotaktische Verbindung der 
Zeichen, ebenso die Abhandlung über den Begriff Translativ als Zeichen dieser sprachlichen Erscheinung. (Darüber wurde von mir in einem Artikel in der kroatischen Zeitschrift Jezik 1959/60 berichtet.)

Kritisch vorgestellt wurde Tesnières Valenztheorie in einer monographischen Bearbeitung der Rektion des Verbs in der slowenischen Schriftsprache (20. Jahrhunderts) von J. Dular (1982). Tesnière wird hier als der Begründer dieses Begriffs und der auf ihm beruhenden Theorie angeführt. Leider ist das Stichwort valenca/vezljivost (vezljiv) in der erwähnten Enzyklopädie (1992) anders bearbeitet.

Aus heutiger Perspektive ist der sprachwissenschaftliche Gedanke Tesnières besonders in zweifacher Hinsicht bedeutend: die Tendenz zur kohärenten Darstellung des Gegenstandes der Syntax und die Methode, nach der das sprachliche Material aus dem Redefluß heraus analysiert wird.

Die Methode der syntaktischen Analyse von Tesnière war schon in ihren Anfängen (1953) und später (1959) auf der geschlössenen Theorie gegründet, die vom Autor durch die Definition von syntaktischen Erscheinungen und die anschauliche graphische Darstellung übersichtlich vorgestellt wurde. Im Ausgangspunkt steht die Konnexion (= Verbindung) zwischen Wörtern als die grundlegende syntaktische Eigenschaft, "ohne die die Phrase (= die Satzverbindung, der Satz) nicht möglich wäre", und der Tesnière die Strukturfunktion zuschrieb. Dieser Erscheinung ist das ganze erste Kapitel des Versuchs, grundlegende Probleme der strukturellen Syntax darzustellen, gewidmet. Die durch Konnexion verbundenen Elemente nach dem Beispiel Alfred poje - Alfred und poje nannte er Nuklei (Kerne) und er schrieb ihnen eine semantische Funktion zu. Laut dieser Theorie (und mit Graphs dargestellt) war der obere Kern leitend (= régissant), der untere Kern war dem leitenden untergeordnet. Laut dieser Theorie ist der Ausdruck im Präclikat, sei es verbal oder verbal-nominal, dem Subjekt übergeordnet: da eine solche Lösung trotz der Satzbildungsfähigkeit des Prädikats nicht gänzlich entsprechend ist, ist verständlich, daß sich in der slowenischen Analyse die Dependenztheorie über die Interpretation in der tschechischen funktional-strukturalen Syntax, die fast gleichzeitig mit der Tesnièreschen entstand (Bauer 1952, Hausenblas 1958), behauptete. Wenn es mehrere Nuklei gibt, sind zwei Konnexionen vorhanden und es entsteht ein Knoten (nœud), der Hauptkern hat die Knotenfunktion und verbindet die Wortverbindungen in eine Struktur, die graphisch durch ein Stemma abgebildet wird. Durch diesen Begriff wird die Phrasenstruktur als Konnexionarchitektur definiert, die Aufgabe der strukturellen Syntax ist es aber, diese Architektur als Eigenschaft einer tiefen strukturellen Realität zu erforschen, die hinter der linearen Reihenfolge einer Sprechkette steckt. Das Stemma als Ergebnis der strukturellen Analyse hat eine angedeutete lineare Struktur, wenn das Verb je (ist) laut heutigen Begriffen eine Valenz besitzt, oder eine zweigliedrige bzw. dreigliedrige Struktur bei zweifacher bzw. dreifacher Verbvalenz. Zwischen den Wörtern im Sprechfluß (Sprechkette), in der Fachliteratur auch unmittelbare Syntaktanten genannt, verläuft die Konnexion nicht immer unmittelbar, deswegen stellt die strukturelle Methode durch das Bloßstellen der 
Verbindungen innerhalb des Stemmas auch die semantische Analyse dar, die nach Tesnière gleichzeitig die bisherigen Verfahren, die grammatische und logische Analyse, ersetzt. Die strukturelle Methode eignet sich auch dazu, strukturelle Verwandschaften von Sprachen miteinander zu vergleichen, es zeigt sich jedoch bei Tesnièreschen Vergleich zwischen Französisch und Englisch mit der Bestimmung des Stemmas im Syntagma nach der linearen Reihenfolge aus der Sprachkette (chien - blanc : dog white) ziemlich deutlich, wie das Slowenische zu ganz anderen Sprachen gehört mit seiner komplizierten semantisch-syntaktischen Struktur (bel-i - pes $+o$ ), bei der die semantische Bestimmung in einem solchen Syntagma in einer Richtung vom Attribut zum Substantiv verläuft, in entgegengesetzter Richtung vom Substantiv zum Adjektiv aber die strukturelle Bestimmung: die erste zeigt sich in der Bedeutung der Verbindung, die zweite in der kongruierten Form des Adjektivs.

Die Tesnièresche Methode blieb trotz der Ausgangsbestimmung der strukturellen Syntax als ein besonderes Gebiet und der Definition der Grundbegriffe nicht bei den sg. Grundstrukturen, d. h. reinen Strukturen; der Gegenstand der strukturellen Analyse ist die innere Struktur der Aussage im Text. Gerade deswegen geriet er in seiner Bestimmung von Spracherscheinungen bald an die Schwelle der Textgrammatik.

Die Begriffe, die er bei der mit Stemma beschriebenen Analyse darstellt, sind zuerst in den Kapiteln über den einfachen Satz erfaßt (der slowenische Terminus "freier Satz" scheint nach einer Überlegung eine leere Vereinfachung, die erst aufgrund der Definition eine Vorstellung ermöglicht); darunter ordnet er auf erster Stelle das Verb als den Knoten der Knoten, ihm untergeordnet sind zuerst Aktanten (an der Handlung beteiligt) und zwar der erste (das Subjekt), der zweite (das Akkusativobjekt) und der dritte (das Dativobjekt oder eventuell das Objekt in anderen Kasus). Es wird besonders hervorgehoben, daß auch das Subjekt untergeordnet ist, was die Analyse von Sprachen slowenischen Typs - wie schön erwähnt - wegen der Kongruenz des Prädikats mit dem Subjekt nicht bestätigt, und so scheint die Tesnièresche Lösung auch hier nur mehr oder weniger eine formalistische Interpretation der ausgewählten graphischen Darstellung der Struktur. Auch auf einer anderen Stelle (Tesnière 1953: 8) wird er bei der Interpretation einer verbal-nominalen Verbindung als eines komplizierten Nukleus (Beispiel davon später bei der Erörterung des Tesnièreschen Definition vom Wortmaterial), in wortwörtlicher Übersetzung Adjektivattribut genannt, auf der führenden Rolle des Prädikatsteiles bestehen, der immer dem Subjektsubstantiv übergeordnet ist. Eine interessante methodologische Anregung kann heutzutage noch ein strukturanalytischer Vergleich zwischen Sprachen unter Feststellung von verschiedenen Stufen der Aktanten beim englischen I miss you und der französischen Wendung mit der ungefähr ähnlichen globalen Bedeutung Vous me manquez, die aber auch in Bezug auf die Bedeutung der Konstituenten so auseinander sind, daß es fraglich ist, ob ein derartiger Vergleich der Strukturtypen sinnvoll bzw. entsprechend ist.

Dem Verbe im Prädikat sind untergeordnet auch Zirkumstanten (Toporišič 1992: 161) gibt den Synonym okoliščina an, das aber nicht der Tesnièreschen strukturellen 
Analyse des konkreten Textes entspricht, sondern es umfaßt die beschriebenen Elemente in der semantischen Grundlage des Satzes) d. h. Ausdrücke, die Umstände der (verbalen) Handlung in Bezug auf Zeit, Ort, Art und Weise usw. bestimmen. Tesnière ist der Meinung, daß die Anzahl der Zirkumstanten unbeschränkt sei. Im Stemma, d. i. verbundenen Strukturstemma, sind die Beteiligten immer rechts von den Aktanten; dabei ist anzumerken, daß die Konnexion immer zwischen dem Verb und jedem Aktanten bzw. Zirkumstanten getrennt verläuft.

Beispiel:

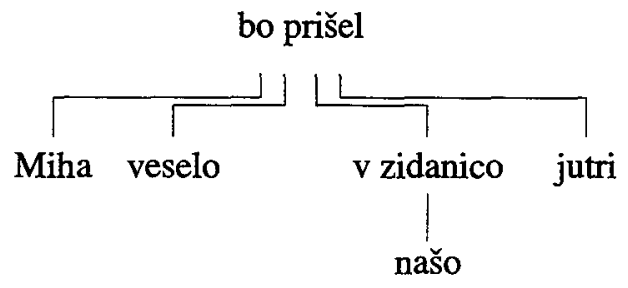

Dem Substantiv untergeordnet ist das Attribut, das Epitheton, das wiederum dem Zirkumstanten übergeordnet sein kann, wenn dieses es näher bestimmt.

Unsere Aufmerksamkeit soll der Abhandlung der Apposition gewidmet werden, von der Tesnière behauptet daß sie eine horizontale Konnexion habe, und dadurch unterscheidet er sie vom Epitheton, das dem Bezugswort untergeordnet ist. Da es bei Stemmen des einfachen Satzes um das einzige Beispiel der horizontalen Konnexion geht, kann man darin verstehen den Strukturunterschied zwischen dem einfachen Epitheton und der Apposition als der im Französischen grammatikalisierten rhetorischen Figur (nach anderen Theorien ist auch die Apposition dem Bezugswort untergeordnet). Genauso ist bemerkenswert, daß die Apostrophe als der erste Aktant des Verbs im Imperativ definiert ist.

Im dritten, vierten und fünften Kapitel (Tesnière 1953) wird die enge Problematik erfaßt, die mit dem Begriff Konnexion als der Grundeigenschaft der Verbindung zwischen den Wortnuklei verbunden ist. Dabei reicht Tesnière zweimal über die Grenze des einfachen Satzes (Phrase) auf den Bereich der Textstruktur, und das zuerst bei der Gliederung der Fragen und im 4. Kapitel, in dem der Wortschatz behandelt wird, im Unterkapitel über die Anapher. Die Fragen teilt er typologisch zuerst auf Kernfragen, das sind solche, die nach Kernen fragen, also Nukleusfragen (nucléaire). In diesen Arten von Fragen wird mit leerem Nukleus gefragt, die Antwort enthält jedoch den entsprechenden vollen Nukleus. Es kann so viele Fragen geben, wie es Nuklei im Satz gibt: leere Wörter sind Fragewörter, die für jede Art der Satzelemente besonders bestimmt sind; nach dem führenden Kern im Prädikat wird mit dem Fragewort und einem Verb mit allgemeinen Bedeutung gefragt (Was macht er?). Meiner Meinung nach wird mit solcher Interpretation der Frage auf den Bereich der Textgrammatik übergetreten, da zum ersten Mal zwischen einer durch ein leeres Wort gebildeten Frage und der durch ein volles Wort gebildeten Antwort eine Art semantischen Konnexion 
entsteht, über die Tesnière bei der Anapher spricht. - Eine andere Art von Fragen stellt nach Tesnière die Konnexionsfrage dar, bei der nicht mehr nach diesem oder jenem Nukleus gefragt wird, sondern nach der Konnexion zwischen ihnen. Die positive Antwort besagt, daß eine Konnexion vorhanden ist, die negative Antwort bedeutet, daß es keine Konnexion gibt. (Die erste Art von Fragen werden traditionsweise auch in der slowenischen Syntax Ergänzugsfragen genannt, die anderen aber Entscheidungsfragen oder Ermittlungsfragen. Keiner von dieser Termini ist vom Standpunkt der strukturellen Syntax konsequent, vor allem die Terminologie der zweiten Gruppe wird nach einer kommunikativen Absicht und nicht nach der Struktur bestimmt.) Aber auch bei der Tesnièreschen Benennung der Konnexionsfrage gibt es eine gedenkliche Inkonsequenz: es gibt eine Konnexion zwischen den Strukturteilen sowohl in der Aussage als auch in der Frage, die positive oder negative Antwort kann also nicht der Feststellung über die vorhandene Konnexion dienen, sondern höchstens über ein spezielles Verhältnis des Sprechers zum Inhalt der Frage bzw. über seine Vermutungen in diesem Zusammenhang. Angesichts dieser Tatsachen erweist sich die Tesnièresche Methode als unzureichend.) - Ähnlich wie die Frage wird auch die Negation gegliedert: auf nukleare (= teilweise), oder konnexionale, es gibt dabei jedoch keinen ausführlicheren Kommentar, wie das der Fall bei der Konnexionsfrage ist.

Die klassische Klassifizierung der Wortarten auf neun oder zehn wird von ihm abgelehnt, aber eine ähnliche Ablehnung war damals seit einigen Jahren aus einer umfangreichen Diskussion über die Wortarten bekannt (Ch. Bühler 1933, J. Kurylowicz 1936 und dort zitierte Literatur). Die Tesnièresche Klassifizierung im behandelten Schema ist als die Konsequenz dieser Ideen einfach: Volle Wörter bilden das Basis-Viereck, in dem das Substantiv und das Verb oben angeführt sind, unter das Substantiv wird das Adjektiv gestellt, daneben steht unter dem Verb das Adverb. Neben ihnen sind leere Wörter, die entweder internukleare Junktive oder intranukleare Translative (= Präpositionen und Subjunktoren) sind. Die dritte Wortgruppe bildet eine besondere Gruppe leerer Wörter, s.g. Anaphoriker, durch welche die Anapher realisiert wird. Diese Wörter sind leer nur im Wörterbuch, in der Phrase haben sie jedoch volle Bedeutung, weil sie durch die Bedeutung eines vollen Wortes gefüllt werden, mit dem sie die anaphorische Konnexion verbindet. Alle Arten der strukturellen Konnexion enthalten gleichzeitig auch die semantische Konnexion dar, nur die Anapher stellt eine zusätzliche semantische Konnexion dar, die keine parallele strukturelle Konnexion hat. Die Tatsache, daß es in einer Phrase mehrere Anaphern geben kann, wird durch einen komplizierten Graph dargestellt, in dem mittels eines unterbrochenen Strichens verschiedene anaphorische Konnexionen besonders hervorgehoben werden. (Beispiel: Kovček moje tete boste izročili njenemu možu in njegov ključ dali njunemu sinu. Sie werden den Koffer meiner Tante ihrem Mann überreichen und seinen Schlüssel ihrem Sohn.) Obwohl hier alle Beispiele innerhalb des einfachen Satzes angeführt werden, geht es bei der Anapher um eine bedeutende Erscheinung in der Transphrasenstruktur, im Text also, wo die Tesnièresche Definition noch immer aktuell ist. 
In das Kapitel über Wörter wurde dann noch die Erklärung über einen zusammengesetzten Nukleus aufgenommen, der überall dort vorkommt, wo zwei Wörter in ein Ganzes zusammengesetzt sind und dieselbe syntaktische Aufgabe haben; das sind vor allem alle zusammengesetzten Verbformen (Tesnière 1953: 8), deswegen sind die Graphe für Alfred poje (Alfred singt) oder Alfred je pel (Alfred hat gesungen) gleich. In dem zusammengesetzten Nukleus je pel (hat gesungen) bildet das Hilfsverb das strukturelle Zentrum, das Partizip ober das semantische: das Hilfsverb ist ein leeres Wort und somit nach Tesnière ein Translativ. Der her zum zweiten Mal erwähnte Begriff des Translativs deutet an, daß Tesnière mit dieser Bezeichnung verschiedene Erscheinungen der Bedeutungsübertragung über bestimmte Struktureigenschaften deckt. Neben der oben erwähnten Darstellung der verbal-nominalen Struktur (diese wird auch in der slowenischen Syntax berücksichtigt werden müssen, die sich bei der Erörterung dieser Frage wenigstens in einem Teile nach der formalistischen und nicht nach der strukturellen Analyse richtet, vgl. [Toporišič 1992: 200] - Stichwort povedkovnik / predikativ oder [Slovenski pravopis 1990: 106, 206] — Stichwort povedkovnik) schließt Tesnière mit dem Definieren der Interjektionen als Phrasenwörter (mot-phrases), d. h. Wörter, die Phrasen äquivalent sind. Er klassifiziert sie in logische (darunter kann die slowenische Partikel — členek [Toporišič 1965, 1985] oder členica [Pogorelec 1961] genannt) und affektive, zu denen er Interjektionen zählt.

Die Konnexions- und Stemmaproblematik wird durch die Gliederung der Verbalvalenz abgeschlossen, die in der slowenischen Syntax heute ein gängiger Begriff ist. Erwähnungswert ist die Gliederung der bivalenten Verben hinsichtlich des Genus auf aktive, passive, reflexive und reziproke Verben (Beispiel: Alfred und Bernard se tepeta = Alfred und Bernard schlagen sich, das ist gegenseitig - oder - Alfred se tepe $=$ Alfred schlägt sich $=$ sich selbst). Bei trivalenten Verben erwähnt er eine komplexe Struktur, viervalente Verben werden von Tesnière nicht anerkannt. Die Behandlung von Valenzvariationen durch Erweiterung bzw. Verminderung der Aktantenzahl greift wiederum in die Grundlagen der Textsyntax ein.

Der zweite Teil ist der Junktion gewidmet, der vollen und partiellen. Die Junktion, das ist die koordinierende Verbindung zwischen den Nuklei in der Phrase (darum ist

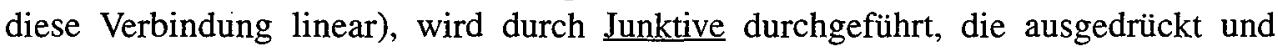
internuklear sind oder nicht ausgedrückt sind (wenn sich um ein Asyndeton handelt). Die Junktive führen die strukturelle Junktion durch und drücken semantische Relationen aus, sie sind jedoch sehr bedeutende Phrasenindikatoren, wenn sie am Anfang einer Phrase stehen. Obwohl auch hier Tesnière nicht über den Text spricht, kann diese Feststellung als ein Hinweis auf die Rolle des Junktivs als eines phrasen übergreifenden Verbinders (Konjunktors) verstanden werden. Verschiedene Bedeutungen der Junktive werden als semantische Varietäten dargestellt, zu denen er folgende zählt: kopulativen Junktiv ( in - und), negierten kopulativen Junktiv (niti . . niti weder . . . noch), disjunktiven Junktiv (ali . . ali - entweder . . oder), adversatives toda (aber), explikatives (kausales) kajti (denn), konsekutives torej (also). Die Junktion 
entsteht zuerst bei der Verdoppelung zweier Aktanten in der Phrase, die durch die Addition zweier Phrasen mit gleichen Aktanten entsteht. (Alfred in Bernard sta padla. - Alfred und Bernard sind gefallen.) - Hier werden noch verschiedene mögliche strukturelle Junktionsvariationen gegliedert, einige Verflechtungen (plexus) werden graphisch dargestellt sowie Vergleichsphrasen, das sind Phrasen bei denen die Junktion zwischen zwei Komparationsausdrücken auftritt: der Junktiv ist kakor, kot (wie, als). In der slowenischen Syntax sind diese Strukturen als untergeordnete analysiert. - Die Junktion ist bei Tesnière integral und partiell, die Beispiele umfassen teilweise den Unterschied zwischen der strukturellen Zusammengehörigkeit und der semantischen Inkompatibilität. - In dem Zusammenhang behandelt er als eine syntaktische Erscheinung auch eingeschobene Phrasen (phrasen incises), die mit der Phrase, in die sie eingeschoben werden, kein strukturelles Band verbindet außer vielleicht eine Spur einer Anapher.

Der dritte Teil ist der syntaktischen Erscheinung gewidmet, die von Tesnière Translation genannt wurde; eine Erscheinung, die man als Transfer (prenos) oder Umsetzung (prestavitev) eines Wortes aus einer grammatikalischen Kathegorie in die andere nennen, und damit die Veränderung seiner ursprünglichen Funktion feststellen könnte. Elemente der Translation sind: der transferend, das ist der zu transferierende Nukleus, der Transferierte ist der Nukleus nach dem Transfer. Das Ausdrucksmittel des Transfers ist der Translativ, der immer bei dem Transferenden ist (meistens als Präposition, in der slowenischen Sprache auch als Morphem der Translation) und intranuklear (intranucléaire) ist. Eine einfache Translation ersten Grades enthält folgende Transfers: die Adjektivisierung des Substantivs und die Substantivisierung des Adjektivs, besonders bei der Pronominalisierung. Der Transfer erfolgt weiter vom Adjektiv zum Adverb und umgekehrt, und auch vom Substantiv zum Adverb, wobei der Translativ eine Präposition ist, weiter vom Verb zum Substantiv bzw. zum Adjektiv. Eine komplexe Translation ersten Grades enthält mehrere Arten von so genannten doppelten Translationen mit verschiedenen Strukturen. Satzgefüge gehören in die dritte Gruppe einfacher Translationen ersten Grades, aber auch komplexer Translationen zweiten Grades. Der Translativ ist ein subordinierendes Bindewort. Die Klassifizierung dieser Bindewörter unterscheidet sich von den klassischen, besonders erwähnungswert ist die Behandlung des Relativs (was ein Problem der Textgrammatik ist).

Die genaue Tesnièresche Behandlung der Translationstheorie ermöglichte zwar die strukturelle und teilweise auch die semantische Analyse und damit die Beschreibung einer ganzen Reihe von Strukturen, am meisten im Graph, sie konnte jedoch im Bereich der tschechischen Syntax, die in den 60er Jahren die stärkste Wirkung auf den slowenischen syntaktischen Gedanken ausübte, nicht einmal terminologisch Fuß fassen (trotz der Erwähnung des Terminus Translativ bei Pogorelec 1964). Durch diese flüchtige Darstellung wollte ich nur auf die heute aktuellen Eigenschaften des Tesnièreschen Syntax errinern: auf sein Bestreben nach einer linguistisch konsequenten und übersichtlichen Beschreibung der Phänomene, auf die Schärfe seiner Beobachtung 
und auf seinen mutigen Versuch, das Komplexe darzustellen ("zu begreifen"). Wie Tesnière seine Theorie aus der zeitgenössischen neueren linguistischen Diskussion ( $\mathrm{z}$. B.: die Wortartentheorie) gebildet hat, sollte man auch seinen Weg zur eigenen Theorie unter die Lupe nehmen (auch mit dem Vergleich der syntaktischen Theorie von R. F. Mikuš 1946, 1952) wie auch spätere Autoren der Tesnièreschen und anderen Richtungen; und bei der immer größer werdenden Einrichtung in die Textsyntax sollte man herausfinden, welcher Teil von Tesnièreschen Ansichten uns bei deren Bildung am meisten helfen kann.

\section{Literaturverzeichnis}

Bauer 1952: J. Bauer, Několik poznámek o pojmech slovní spojení, větná dvojice a syntagma. V: Sbornik prací FFBU, A I. Brno. 26-57.

Bühler 1934: K. Bühler, Sprachtheorie. Die Darstellungsfunktion der Sprache. Jena. Verlag von Gustav Fischer.

Dular 1982: J. Dular, Priglagolska vezava v slovenskem knjižnem jeziku (20. stoletja). Disertacija. Filozofska fakulteta v Ljubljani. Rokopis. Strani 259.

Hausenblas 1958: K. Hausenblas, Syntaktická závislost, způsoby a prostředky jejîho vyjadrování. V: Bulletin Vysoké školy ruského jazyka a literatury II. Praha. 3-31.

Kaučič-Baša 1982: M. Kaučič-Baša, Rodilnik zanikanja. Slavistična revija. Letnik 30 (1982), št. 3. Str. 305-321.

Kuryłowicz 1936: J. Kuryłowicz, Dérivation léxicale et dérivation syntaxique. (Contribution a la théorie des parties du discours). V: B.S.L. XXXVII, 79-92.

Križaj-Ortar 1985: M. Križaj-Ortar, Vezljivost samostalniške besede. Magistrska naloga. Filozofska fakulteta v.Ljubljani. Rokopis. Str. 109.

Mikuš 1948: R.F. Mikuš, En marge du sixième congrès international des linguistes (Paris 1948).

Mikuš 1952: R.F. Mikuš, A propos de la syntagmatique du professeur A. Belić. K sintagmatiki prof. A. Belića. Slovenska akademija znanosti in umetnosti. II. razred. Dela št. 5. Ljubljana.

Orešnik 1992: J. Orešnik, Udeleženske vloge v slovenščini. Slovenska akademija znanosti in umetnosti. II. razred. Dela št. 37. Ljubljana.

Orešnik in Perlmutter 1973: J. Orešnik in D.M. Perlmutter, Razlaganje sintaktičnih posebnosti. Ljubljana. Institut Jožef Štefan. Poročilo P-282.

Pogorelec 1959: B. Pogorelec, Začetni problemi sistematičnega raziskovanja slovenske sintakse. Jezik 1959/60. Zagreb. 143-155.

Pogorelec 1964: B. Pogorelec, Veznik v slovenščini. Disertacija. Filozofska fakulteta v Ljubljani. Str. 561. 
Sgall, Hajičova, Panevova 1986: P. Sgall, E. Hajičova, J. Panevova, The Meaning of the Sentence in Its Semantic and Pragmatic Aspects. Czechoslovak Academy of Sciences. Prague.

Slovenski pravopis 1990: Slovenski pravopis. 1. Pravila / [uredniški odbor Jože Toporišič et al.] Slovenska akademija znanosti in umetnosti. Ljubljana. DZS.

Tesnière 1953: L. Tesnière, Esquisse d'une syntaxe structurale. Paris. Klinksieck.

Tesnière 1959: L. Tesnière, Eléments de syntaxe structurale. Paris. Klinksieck.

Toporišič 1982: J. Toporišič, Nova slovenska skladnja. Ljubljana. DZS.

Toporišič, 1984: J. Toporišič, Slovenska slovnica. Druga izdaja (pregledana in razširjena). Maribor. Založba Obzorja.

Toporišič 1992: J. Toporišič, Enciklopedija slovenskega jezika. Ljubljana. CZ.

Vidovič-Muha 1988: A. Vidovič-Muha, Slovensko skladenjsko besedotvorje ob prime-

rih zloženk. Ljubljana. Znanstveni inštitut Filozofske fakultete (in Partizanska knjiga).

Povzetek

TESNIÈRJEVA ODVISNOSTNA SLOVNICA IN NOVA SLOVENSKA SINTAKSA

Znanost o slovenskem jeziku je bila $\mathrm{v} 20$. stoletju, zlasti po 1919 , naravnana sprva $\mathrm{v}$ raziskovanje zgodovinskih pojavov $v$ jeziku, slovenske lingvogeneze in $v$ oblikovanje zgodovinske slovnice, ki se je ustavilo pri opisu zgodovinskega glaslovja in naglasnega sestava, deloma oblikoslovja in dialektov, ki naj bi predstavljali nasledek zgodovinskega razvoja slovenskega jezika. Raziskovanje sočasnega slovenskega, predvsem knjižnega jezika, posebej še njegove skladnje, se je le občasno pomudilo ob posameznih izbranih jezikoslovnih problemih. Tako je bila med prvimi razmeroma zgodaj predstavljena funkcijska teorija besednega reda $v$ povedi oziroma $v$ besedilu (Breznik: 1908), in sicer s pojasnitvijo nevtralne naravnanosti perspektive povedi na novo, to je na aktualno. Teoretski okvir za druga vprašanja slovenske sintakse je v tem času mogoče razbrati iz poglavij o sintaksi v srednješolskih normativnih slovnicah (zlasti Breznik ${ }^{1}$ 1916, ${ }^{4} 1934$ ). Poleg tradicionalnega formalističnega pristopa je mogoče $v$ obdelavi nekaterih problemov opaziti nagnjenost $\mathrm{k}$ funkcijski analizi jezikovnih pojavov. Ta naravnanost seveda ni temeljila na kaki bolj ali manj konsistentni teoriji, ki bi enovito zajela pojave na skladenjski ravnini.

Teoretsko izhodišče za sodobno pojmovanje sintakse v smislu razumevanja jezika kot hierarhično urejenega sistema znamenj, kakor ga je pobudil de Saussure, je v slovenskem jezikoslovju tega časa izdelal romanist $R$. F. Mikuš (že okrog 1948, posebej 1952) s svojo sintagmatsko teorijo, v kateri je sistematično, od najmanjše skladenjske enote do zapletene zveze sklenjeno predstavil svoje pojmovanje skladenjske zgradbe in razmerij $v$ njem.

Mikuševa teorija v svojem času v slovenskem prostoru ni mogla odmevati, ker se sočasno jezikoslovje v Sloveniji $z$ moderno jezikoslovno teorijo ni ukvarjalo, bodisi ker so se nadaljevale raziskave historične slovnice in dialektologije, začete pred drugo svetovno vojno, ali pripravljala dela, namenjena jezikovnemu poučevanju.

Prve nove raziskave slovenske sintakse pa so se nekoliko kasneje že oprle na odvisnostno (dependenčno) sintakso, vendar ne neposredno na Tesnièrjev koncept, ampak predvsem na teorijo, kakor jo je kot odmev na sočasno diskusijo o sintaksi v svetu razvilo češko jezikoslovje (zlasti Bauer 1952, Hausenblas 1958 in drugi). Ne nepomemben razlog za ta vpliv je $v$ jezikovni sorodnosti slovanskih jezikov, ki se po izrazilih razlikujejo od jezikov, ki jih je za svojo abstraktno shemo uporabil Tesnière. Zato Tesnièrjeva strukturalna odvisnostna teorija ni vplivala na novo slovensko jezikoslovje $v$ svoji koherentni podobi, ampak prek posrednikov ob obravnavi posameznih skladenjskih vprašanj. Tako je bila ta teorija eno od izhodišč za obdelavo veznika v slovenščini (Pogorelec 1964), za formuliranje osnovne analize (pre)prostega stavka (Toporišič 1965), nadrobneje prikazana je 
bila tudi $\vee$ študiji o priglagolski vezavi $v$ sodobnem slovenskem knjižnem jeziku (Dular 1982). To je bil nemara razlog, da tudi v Enciklopediji slovenskega jezika Tesnièrjeva teorija ni prikazana v svojih poglavitnih spoznanjih (Toporišič 1992).

Danes, ko postaja predmet skladenjskih raziskav predvsem besedilo, kaže, da bo potrebno in koristno tudi v slovenskem prostoru o Tesnièrjevi teoriji v celoti in podrobnostih ponovno razpravljati, posebej še o njegovem pojmovanju anafore kot posebne vrste besedilnega povezovalca. Smisel nove diskusije o Tesnièrju pa se kaže tudi spričo urejene in transparentne metode, ki gradi svoj opis na konkretnih in preverljivih jezikovnih dejstvih. Za nas produktivno je na primer Tesnièrjevo obravnavanje tudi zloženih glagolskih oblik $v$ povedku $v$ njihovi polni in celoviti vlogi, kar bi kazalo $v$ celoti ponovno vpeljati tudi $v$ slovenske analize. 